Revue d'histoire de l'Amérique française

RAS REVUE D.HISTOIRE DE L'AMÉRIQUE FRANÇAISE

\title{
Liberté économique et politique des Canadiens sous le régime français
}

\section{Rosario Bilodeau}

Volume 10, numéro 1, juin 1956

URI : https://id.erudit.org/iderudit/301742ar

DOI : https://doi.org/10.7202/301742ar

Aller au sommaire du numéro

Éditeur(s)

Institut d'histoire de l'Amérique française

\section{ISSN}

0035-2357 (imprimé)

1492-1383 (numérique)

Découvrir la revue

\section{Citer cet article}

Bilodeau, R. (1956). Liberté économique et politique des Canadiens sous le régime français. Revue d'histoire de l'Amérique française, 10(1), 49-68.

https://doi.org/10.7202/301742ar d'utilisation que vous pouvez consulter en ligne.

https://apropos.erudit.org/fr/usagers/politique-dutilisation/ 


\section{LIBERTÉ ECONOMIQUE ET POLITIQUE DES CANADIENS SOUS LE REGIME FRANÇAIS *}

Nous demander si, sous le régime français, les Canadiens ont bénéficié de liberté économique et politique, c'est admettre d'abord que cette liberté puisse exister dans une colonie; et qu'elle puisse y exister même si cette colonie dépend d'une métropole qui vit sous le signe de l'absolutisme royal en politique, du mercantilisme en économie, et qui, dans la mesure du possible, met la politique au service de l'activité économique dirigée. C'est que la liberté nationale n'est attachée à aucune forme de gouvernement. La monarchie centralisée a aussi bien atteint à la grandeur de la France, donc à la liberté possible du peuple français, que le régime parlementaire anglais a atteint à l'empire et, ainsi, à la liberté possible de la nation anglaise. « La politique, affirme Jean Chevalier, n'est que l'art de s'adapter aux circonstances (et) un régime ne s'impose qu'autant qu'il répond à l'orientation des esprits et aux exigences du moment». L'histoire nous l'a appris. La liberté n'est pas non plus liée à un système d'économie puisque l'économie dirigée, aussi bien que l'économie libérale, peut garantir la liberté d'un groupe humain constitué en nation, et que les diverses formes de gouvernement peuvent se superposer à l'économie libérale ou à l'économie dirigée.

Le Canada dont l'histoire s'inscrit dans les cent cinquante années du régime français est une colonie, donc un territoire destiné, selon le contexte historique des $\mathrm{XVI}^{\mathrm{e}}$ et $\mathrm{XVII}{ }^{\mathrm{e}}$ siècles, à enrichir un Etat-nation ambitieux d'accroître sa puissance. Mais cette colonie ne sera pas uniquement un comptoir commercial. La métropole ne l'entend pas ainsi, et les premiers habitants du Canada voudront, en s'y établissant, en faire leur pays, et ils auront, en s'y accroissant en nombre et en richesse, la volonté de diriger ce pays dans le sens de leurs intérêts de groupe.

* Substance d'une thèse présentée à l'Institut d'histoire de l'Université de Montréal pour l'obtention d'un doctorat ès lettres. 
Ainsi, entre le moment de la fondation de la colonie et celui de la Conquête, d'une part, entre les visées mercantilistes de la métropole et la volonté de la société canadienne de diriger ses affaires et son gouvernement, d'autre part, oscille la pratique d'une liberté que la classe dirigeante canadienne s'est plus préoccupée de mettre à profit que de définir. Il s'agissait, pour ce groupe, de vivre, et d'en trouver les moyens avec ou contre la politique du royaume telle qu'exprimée dans les institutions, et d'après la force croissante que le nombre et la richesse lui procuraient selon les moments de son évolution.

Dans une colonie, en effet, dont la structure politico-sociale s'appuie sur le commerce, un groupe dirige les affaires et oriente ou influence la politique dans ce sens. Le Canada était normal et de son temps: au sommet de la société canadienne régnait une grande bourgeoisie dont l'effort pour se hisser et se maintenir à la tête du commerce et de l'administration, en entraînant, consciemment ou non, la collectivité à sa suite, représente la conquête constante de la liberté politique et économique. Ce ne peut être autrement que cette classe, qui donne le ton à la société, entendît la liberté dont nous traitons, et la revue que nous allons présenter de l'actualité passée démontrera que c'est de cette façon que les Canadiens ont voulu la mettre à profit. Nous ne traitons donc pas de liberté individuelle: qui dit liberté économique et politique dit nécessairement liberté de groupe. Et la liberté économique n'existe pas sans la liberté politique, la première étant conditionnée par la seconde. Elles sont inséparables.

C'est ainsi que nous nous attacherons à suivre l'activité économique et politique de la classe dirigeante canadienne dans les divers moments de son évolution. Cette classe se constitue très tôt des seigneurs-négociants, gentilshommes ou roturiers. Les «principales familles » de la colonie, comme on les appelait souvent, unies ou par les liens du sang ou par la communauté d'intérêts et d'idées, s'efforceront de dominer le grand commerce d'alors, celui des fourrures, et feront agir, selon les besoins de ce commerce, le gouvernement colonial où elles seront représentées. 
La France du XVII ${ }^{e}$ siècle et de la première moitié du XVIII siècle est un Etat-nation mercantiliste. Cherchant à constituer sa richesse par l'accumulation de métaux précieux et par un effort conséquent à limiter l'importation et à accroître l'exportation, elle se devait, comme tous les jeunes Etats-nations, de posséder une colonie qui lui procure, sinon des métaux précieux, du moins des objets de commerce ou des matières premières qui permettent de conserver la monnaie dans le royaume. Fondée principalement dans ces vues, la Nouvelle-France devait se développer selon ces principes puisque, durant son existence, elle était dirigée, du moins de loin, et soutenue à des degrés divers, par un Etat mercantiliste.

Les difficultés politiques et financières où se débattait la France l'amenèrent d'abord à confier à des compagnies de commerce l'établissement des colonies. Ces compagnies se rembourseraient des frais de voyage et d'établissement par le privilège exclusif du commerce colonial. Le conflit d'intérêts était inévitable. Champlain voulait créer un établissement, et les détenteurs du monopole s'intéressent avant tout aux opérations de traite. Mais Champlain a la protection de la Cour et il y poursuit ses instances pour qu'elle oblige les marchands à s'occuper du peuplement. En 1621, les quelques « habitués» qui vivent près du comptoir de Québec exposent leurs griefs contre la compagnie dans «le Cahier du Païs » que le Père Le Bailliff présentera à la Cour. Déjà les premiers habitants y cherchent protection. C'est le premier d'une innombrable série de voyages où les Canadiens exposeront leurs vues au roi. Vingt ans après sa fondation, toutefois, Québec ne compte encore que 76 personnes, et c'est le seul poste au Canada. Pourtant, ce poste donne déjà en fourrures jusqu'à 200,000 livres par année. Il est facile de prévoir sur quoi se basera l'économie de la colonie. Ceux qui tiendront la bourse, garnie par les bénéfices du commerce, voudront administrer la chose publique selon les nécessités économiques.

Le cardinal de Richelieu savait que les marchands n'avaient à peu près rien fait pour le peuplement. S'il confie ce soin aux Cent Associés, c'est qu'il sera l'âme dirigeante de la Compagnie. 
Mais dès le début de ses opérations, la nouvelle société subit des pertes qui s'élèvent à environ 300,000 livres. L'effort ne sera repris qu'à la fin des hostilités. Après 1635, il passe au Canada de quinze à vingt personnes par année. Richelieu ne retira pas des compagnies des bénéfices proportionnés aux privilèges qu'il leur accordait. On a pensé, pour remédier à cette insuffisance, à attirer dans la colonie des hommes riches et entreprenants qui puissent se charger de peuplement. Ainsi furent faites les premières concessions de seigneuries à partir de 1634 .

Entre le seigneur et le censitaire s'établit un lien de nature économique. «Les terres y sont possédées en seigneuries, tenure selon laquelle plusieurs personnes partagent, à des titres divers, un ensemble de droits et de charges relatifs à une même concession. 》 Les seigneurs concessionnaires, cependant, ne disposant pas, en général, du capital nécessaire à l'exploitation agricole, manquant d'expérience et ambitieux de revenus plus faciles et considérables, se lancent pour la plupart dans le grand commerce de la colonie, la traite des fourrures. Avec ce groupe de seigneurs-négociants naît la première oligarchie canadienne. Dès lors, elle n'attend que l'occasion de s'emparer du monopole de la traite.

Les autorités métropolitaines, mécontentes des déficiences de la Compagnie à l'égard du peuplement, seraient attentives à toute proposition nouvelle. Les seigneurs-négociants, qui forment déjà une classe homogène, bien que très peu nombreuse, demandent que la traite soit remise entre leurs mains en affirmant que c'est le seul moyen d'accroître et d'affermir la colonie. Ils s'entendent avec les Jésuites et la Société Notre-Dame de Montréal, obtiennent ce monopole en mars 1645 , et s'associent sous le nom de Compagnie des Habitants.

Cette compagnie recueille les pelleteries dans la colonie et prélève les droits destinés à solder les frais de l'administration coloniale. Les nouveaux maîtres du commerce intérieur s'assurent l'appui, sinon le contrôle, de l'administration politique qu'ils alimentent des revenus de leur commerce. A partir de 1647, ils dominent les conseils dont le gouverneur s'entoure. Ainsi, s'appuyant l'un sur l'autre, vont se développer simul- 
tanément le commerce des fourrures et les rouages administratifs. Le même facteur, le grand commerce, domine la vie économique aussi bien que l'activité politique. Ceux qui sont à sa tête seront aussi à la tête de la société canadienne. Tel est le centre nerveux de tous les efforts. Tel est du même fait, le dénominateur qui nous permettra d'établir la mesure de liberté politique et économique dont jouira la classe supérieure au Canada durant le régime français. Chaque fois que la bourgeoisie canadienne contrôle le gouvernement colonial et dirige le grand commerce, elle jouit de toute la liberté qu'une colonie puisse posséder. Quand elle est délogée, nous la voyons faire pression de tous côtés pour se hisser de nouveau à cette direction.

Dès leur nomination, les conseillers se votent des gratifications que M. de Maisonneuve trouve exorbitantes; il y eut contestations. Les «principales familles » obtiennent gain de cause. C'est que cette classe jouit de la suprématie dans la colonie, et tous les administrateurs métropolitains devront compter, sinon sur une alliance avec elle, du moins sur sa présence active. Le rôle du Conseil, instrument d'influence de la bourgeoisie, est de régir l'administration intérieure de la colonie et de rendre la justice. La métropole dirige la politique extérieure. Les décisions relatives à la guerre contre les colonies anglaises, par exemple, relèvent du royaume. Mais quand les objectifs des Canadiens l'exigent, ceux-ci peuvent déjà faire pression auprès du roi pour qu'il fasse la guerre et envoie des secours. La politique intérieure, par contre, est plus directement influencée par les «habitants ». Le gouverneur est président du Conseil, mais il ne peut pas se permettre d'ignorer le Conseil. Si le gouverneur participe au commerce, il doit s'allier à l'oligarchie canadienne, sinon il provoque un commerce clandestin quand il ne hâte pas son propre rappel. Les Canadiens possédaient alors un gouvernement équivalant presque à l'indépendance, un «self-government », dans le cadre de l'empire français.

Durant cette période qui va de 1645 à 1665 , le mercantilisme métropolitain a favorisé le commerce canadien. La France fournit un marché assuré à sa colonie. Mais les bénéfices du commerce ne furent cependant pas au niveau des espoirs de la 
bourgeoisie. La Compagnie s'endette pendant que les individus s'enrichissent. Les guerres incessantes des Iroquois nuisaient aux communications et empêchaient les Hurons de descendre à Montréal et à Québec. Il n'y a alors que l'Etat métropolitain qui puisse intervenir pour protéger le commerce, cette tâche étant bien au-dessus des moyens de la petite colonie. Les seigneursnégociants, par leurs appels au Conseil et leur influence auprès du gouverneur, amènent finalement le roi à expédier des troupes dans la colonie. Tracy rétablit la paix. Elle était nécessaire à la prospérité du commerce.

A ce moment, Jean Talon devient intendant. Cette nouvelle fonction vise à maintenir l'équilibre entre les pouvoirs du gouverneur et ceux du Conseil souverain. Le Conseil souverain, créé en 1663, visait à établir une justice réglée pour tous les coloniaux. Pressé d'activer le peuplement, Talon distribue à de nombreux officiers du régiment de Carignan des seigneuries aux endroits stratégiques de la colonie. Quelques centaines de soldats s'établissent sur des terres. L'intendant «place ainsi, à côté de la première équipe de colonisateurs, un autre élément auquel la bourgeoisie n'ouvre pas volontiers ses cadres ». Les nouveaux seigneurs rechercheront les postes dans l'administration, le commandement de stations militaires, et quelques-uns s'adonneront plus spécialement au commerce. Ils formeront peu à peu, avec l'ancienne aristocratie, la classe dirigeante du début du XVIII ${ }^{e}$ siècle.

Mais le premier souci des «habitants » est de conserver le monopole de la traite. Louis XIV l'avait octroyé à la Compagnie des Indes Occidentales en 1664. C'était, pour la bourgeoisie canadienne, une menace de ruine. Aussi, l'année suivante, revenait-elle en place. Talon obtint que les «habitants» soient admis à faire la traite en même temps que la Compagnie et sur un pied d'égalité avec elle. La Compagnie disparut au bout de quelques années. Le commerce intérieur reste toujours, de droit ou de fait, aux mains des classes dirigeantes de la colonie. Colbert hésitait à accorder cette liberté par crainte que les «habitants» ne demeurent oisifs durant une partie de l'année et qu'ils ne cultivent pas leurs terres. Mais si les «habitants » 
ne peuvent vivre, ils retourneront en France. «Talon voit justement dans la traite la seule activité capable de retenir dans la colonie une bourgeoisie que l'agriculture ne saurait intéresser. »

Dès son arrivée, d'ailleurs, l'intendant avait pris parti pour les marchands canadiens contre les agents de la Compagnie des Indes Occidentales. Il ne voulait pas que le Canada soit regardé comme un simple lieu de commerce mais comme un pays appelé à devenir une puissance. Il fait appel au roi pour aider le pays dans ses commencements. Colbert voulait doter la NouvelleFrance d'une économie complémentaire de celle des autres établissements de l'empire colonial français. Mais Talon s'identifie aux intérêts de la colonie. Il travaille à la colonisation intégrale du Canada et non seulement aux industries complémentaires. Ses demandes en faveur de la liberté commerciale, de la hausse du prix des fourrures, son ambition de créer ici un riche royaume, c'est-à-dire un pays qui pourvoie par lui-même à ses besoins essentiels, le stimulant qu'il a donné au commerce d'importation et d'exportation, l'inauguration qu'il a faite d'échanges commerciaux avec les Antilles et les suggestions de trafiquer avec les Anglais par le lac Champlain ou, par la rivière Saint-Jean, avec les Français de l'Acadie qui sont sous leur domination, suffisent à faire penser qu'il est si pleinement entré dans les desseins mercantilistes de Colbert qu'il s'est orienté vers le même mercantilisme en faveur du Canada.

Le mercantilisme métropolitain restreignait la prospérité du commerce et nuisait à la bourgeoisie qui voulait le diriger vers les marchés les plus lucratifs. Talon dut accepter, à la fin, de ne pas «blesser la liberté du commerce qui fait la richesse de la France ». A ce moment, le mercantilisme impose une restriction à la liberté des Canadiens. Remarquons cependant que tout ce qui renforce la France, renforce le Canada. Durant cette période, l'initiative vient d'abord de l'intendant. Aubert de La Chesnaye lui reprochait même d'avoir voulu « réunir le gouvernement avec l'intendance ». Il l'accusait aussi d'avoir fortement concurrencé les marchands. Les marchandises de l'intendant lui arrivaient quittes de droits, de fret et d'assurances. La 
Chesnaye raconte que Talon a refusé de payer l'entrée au pays des vivres, de l'eau-de-vie et du tabac. Il faut noter tout de même que ces accusations viennent d'un des grands marchands canadiens.

Les institutions politiques se sont fixées à peu près définitivement sous Talon. Leur équilibre garantissait, en principe, la liberté des Canadiens. Dans la pratique, tout dépendra des hommes. Louis XIV répète constamment aux administrateurs de maintenir l'union et l'harmonie, et qu'il ne veut rien faire sans le consentement universel de ses peuples. La classe dirigeante représente «l'opinion publique » et agit dans la colonie et à la Cour. L'essor donné par Talon au développement économique de la colonie, essor qui accroissait la liberté du peuple canadien, n'aura malheureusement pas de suites.

Frontenac arrive en 1672. Ruiné de fortune, il s'empresse, grâce au poste qu'il occupe, d'établir un comptoir de traite à Katarakoui, au confluent de la rivière du même nom et du lac Ontario. François-Marie Perrot, gouverneur de Montréal, possédait jusqu'alors, sur l'île qui porte son nom, au confluent de l'Ottawa et du Saint-Laurent, un poste d'avant-garde. Perrot, qui avait concurrencé les principaux marchands de Montréal, se sentit vivement atteint à son tour par l'établissement de ce poste à soixante lieues plus haut que le sien. Frontenac, de plus, disposait à son gré des permissions de traite, le « racket » du temps, et obligeait tous les traitants à s'en procurer de lui. Perrot, pour protéger son commerce, refuse d'obéir au gouverneur général et oblige, lui aussi, les chercheurs de pelleteries à obtenir des congés de traite signés de sa main. Frontenac fit emprisonner Perrot et cita devant le Conseil l'abbé de Fénelon. Celui-ci avait, le jour de Pâques 1674, prononcé un sermon où il traitait, entre autres choses, des devoirs des gouvernants. Le sermon envenima la querelle. Le procès fut porté en France. Les deux concurrents sont bien apparentés. Perrot revient au gouvernement de Montréal, et Frontenac trouve plus prudent de s'entendre avec lui.

Pendant ce temps, La Salle, affidé de Frontenac, obtenait le monopole de la traite du lac Ontario et, plus tard, du lac Michi- 
gan, puis du Mississipi. La grande bourgeoisie se voyait évincée du commerce des fourrures. Frontenac se met de part avec les coureurs de bois qu'il protège, et fait la lutte aux marchands canadiens. Les querelles entre le Conseil et le gouverneur général s'expliquent par cette concurrence dans le grand commerce. Il n'y avait d'ailleurs pas d'intendant pour maintenir l'équilibre entre les pouvoirs.

Duchesneau est nommé à ce poste en 1675 . Les Canadiens trouvent en lui un allié. Duchesneau accuse Frontenac et ses protégés, Perrot, La Salle, Duluth, de commerce interlope, tandis que Frontenac porte la même accusation contre Duchesneau et les marchands canadiens. Il semble que les deux factions aient eu quelque raison. Les pressions de la bourgeoisie canadienne obtiennent le rappel de Frontenac en 1682. Il aurait mieux valu ne pas porter atteinte à la liberté de cette classe. Frontenac, pour la même raison, a failli être rappelé vers la fin de son deuxième gouvernement. Mais l'abolition des congés de traite calma, cette fois, la bourgeoisie.

Ce sont ces mêmes marchands canadiens qui vont aussitôt s'allier au successeur de Frontenac, Lefebvre de La Barre. Celuici se constitua «le premier changeur à la foire aux castors », et s'appliqua à déposséder l'ancienne faction au profit de la nouvelle. On songeait, depuis quelques années déjà, à exploiter la traite de la baie d'Hudson. Le gouverneur convoque les notables de la colonie, et quatorze grands marchands canadiens s'associent pour fonder la Compagnie du Nord. Les associés représentent « les principaux habitants et marchands du pays». La Chesnaye et Le Moyne sont parmi les plus actifs du nouveau groupe. Les fils de Charles Le Moyne, Iberville et Sainte-Hélène, ont à surveiller les intérêts de la Compagnie pendant les campagnes qui se succèdent pour déloger les Anglais de la baie d'Hudson. Le roi recommande (1689) au gouverneur et à l'intendant d'accorder à la Compagnie « une forte protection pour la continuation de leur commerce et pour chasser les Anglais» de ces postes. Ici, les intérêts du royaume et ceux de la bourgeoisie canadienne convergent vers le même but. En 1694, Iberville et Sérigny, son frère, sont les principaux bailleurs de 
fonds de l'expédition qui délogera les Anglais. Deux ans plus tard, Iberville possède le monopole de la traite du Nord. Il le conserve jusqu'en 1700 , alors que de nouveaux arrangements réunissent la bourgeoisie canadienne sous le nom de Compagnie de la Colonie.

La paix que les Canadiens imposèrent aux Iroquois en 1701 a été préparée par la classe dirigeante canadienne qui fournit les principaux agents des négociations. Cette paix ouvrait la voie à l'expansion canadienne vers le Sud et rendait possibles des relations commerciales avec les Anglais. Mais le pacte colonial défendit tout commerce avec les Anglais, le commerce ne devant se faire qu'avec la France. Au moment où le marché français était saturé de castor canadien, cette mesure mercantiliste pèse lourdement sur la vie économique de la Nouvelle-France et présente, du point de vue colonial, une regrettable restriction à la liberté économique et politique. La colonie n'est pas libre de modifier cette ligne de conduite.

En même temps que la Cour imposait cette restriction au commerce extérieur canadien, elle encourageait l'expansion du Canada vers le Sud. Toutefois, ce n'est pas la Cour qui a conçu ce projet; c'est la justesse de vues d'Iberville qui s'est imposée à la Cour. Et ce n'est pas le roi qui va mettre le plan à exécution; c'est Iberville qui en entreprend la réalisation avec l'aide d'une métropole sur laquelle il aurait dû pouvoir davantage compter. Cet événement relève de la politique canadienne de conquête. Le Canada a besoin, par suite des nécessités qu'impose le développement du commerce des fourrures, «d'articuler le bassin du Saint-Laurent au reste de l'Amérique ». Cet impératif explique les conquêtes d'Iberville à la baie d'Hudson, en Acadie, à TerreNeuve et sur le Mississipi inférieur, entre 1686 et 1701 . Le Canada n'est pas libre de ne pas s'étendre: «sa prospérité repose sur la traite des fourrures »; sa classe dirigeante, ne pouvant subsister du produit des seigneuries, «vit du réseau de postes - à la fois comptoirs de traite et stations militaires qui commande les voies de communications de la NouvelleFrance ». Et la Nouvelle-France ne peut se passer de sa bour- 
geoisie: la retraite des principales familles amènerait sa ruine, comme le remarquait Talon.

En somme, c'est la liberté même des Canadiens qui les oblige à ces conquêtes. La liberté économique et politique qu'ils possédaient déjà leur a permis de concevoir nettement, en accord avec la réalité économique où ils vivaient, la politique qu'il leur fallait - et sans cette liberté et sans le sentiment que c'était de leur chose à eux qu'ils s'occupaient, ils n'auraient même pas pu concevoir cette politique - et cette même liberté supposait la puissance de concrétiser cette conception par la réalisation des objectifs visés.

La fondation de la Louisiane, issue de la pensée politique canadienne, nous en fournit la démonstration. La politique réaliste qu'Iberville y a pratiquée, l'exercice auprès des tribus du Mississipi de la diplomatie indigène qui lui avait si bien réussi au Canada, montre que ces Canadiens savent ce qu'ils veulent, et le veulent non seulement avec force mais aussi avec adresse. Iberville a de même compris qu'il fallait conserver deux voies de pénétration en Louisiane, une par le Nord et l'autre par le Sud, et qu'à cette fin le contrôle de la région de l'Ohio était d'une nécessité primordiale. Il posait ainsi les bases permanentes de la politique de la Nouvelle-France en Amérique. Ce qu'on a appelé «le système canadien » est ici tout exprimé. Cette création témoigne donc d'une pensée politique d'envergure, qui sourd des besoins de la vie économique de la colonie. Une telle pensée, nous le répétons, ne peut naître que d'une société - même si les membres de sa classe dirigeante ne sont pas tous capables des mêmes vues - qui jouit d'une liberté de groupe suffisante pour se mettre en frais de dominer tout un continent, affirmant ainsi et sa conception et sa pratique de la liberté économique et politique. Un demi-siècle plus tard, l'enjeu du conflit ne sera pas autre.

Pendant ce temps, au Canada, la bourgeoisie réunie dans la Compagnie de la Colonie, est aussi très active dans l'organisation de la traite. Les circonstances, cependant, ne la favorisent guère. Et après quelques années de lutte, au milieu d'une crise générale, ce sera la faillite. L'effondrement de la Compagnie 
s'explique par certaines maladresses d'administration sans doute; mais plus encore par la saturation du marché français, le seul ouvert à la colonie, par le nombre de postes qu'elle devait exploiter et les grandes dépenses ainsi encourues, par l'état de guerre, par l'opposition que la Compagnie rencontrait au Canada et à la Cour, et par le défaut de capital. La métropole, alors qu'elle aurait dû aider les associés, les contraint au contraire d'établir des accords commerciaux avec des agents français qui exigent un intérêt de 10 p. 100 sur les avances de fonds. Mais le fait significatif, à notre point de vue, c'est que presque toute la bourgeoisie canadienne en fit partie et était consciente, on le voit à la violence des débats quand Raudot démet les directeurs de leurs postes, que c'était sa chose à elle qu'elle dirigeait.

Nous constatons chez les Canadiens la même conscience, la même perspective devant les faits, et la même volonté d'action quand le traité d'Utrecht (1713) démembre la Nouvelle-France en la réduisant à deux petites colonies agricoles, l'une au Sud, l'autre dans la vallée du Saint-Laurent. La métropole n'a pas vu cette conséquence fatale, en accordant aux Anglais le protectorat sur les Iroquois de la région de l'Ohio. Mais les Canadiens n'hésitent pas devant la politique qui s'impose à eux; leur vie économique est menacée; il leur faut éluder les conséquences de ce traité. Aussi vont-ils chercher «à ancrer leur pays au CapBreton, à le prolonger vers l'ouest et à bloquer l'avance britannique sur le Centre-Ouest, ce qui inclut le maintien de la ligne de l'Ohio ». Tel est le fondement de la politique canadienne; et son action procède d'une vue réaliste des exigences économiques. Et voilà que la politique canadienne ne peut s'accorder à celle du royaume tellement les vues diffèrent, et tellement les hommes aussi, pétris dans leur pensée, dans leurs besoins économiques et dans leurs habitudes d'action par cette réalité nouvelle qu'est le Canada, diffèrent des métropolitains.

Le traité d'Utrecht accordait en outre la liberté de commerce aux Indiens. Cette liberté ne peut que favoriser l'Angleterre; la Nouvelle-France ne peut l'admettre à cause de l'avantage économique qu'ont sur elle les Anglais. Elle annule cet article en usant de représailles contre les Indiens qui trafiquent. Elle le 
fait chaque fois que son commerce est en danger de passer à l'étranger. C'est la raison des guerres comme ce fut celle des conquêtes antérieures. Les Canadiens corrompent les sauvages par des présents et font vivre ces tribus sous la menace de la destruction. C'est la même politique qu'en Louisiane.

La crise que traverse la colonie, de 1713 à 1728, s'aggrave de l'état stagnant de l'économie en France. Au Canada, l'intendant Bégon, déjà accusé de se rendre «maître de tout le commerce » - cette accusation vient des négociants canadiens spécule aussi sur les produits agricoles grâce au pouvoir qu'il a de fixer les prix (Bigot ira plus tard jusqu'à contrôler, par une série d'ordonnances, non seulement les prix, mais aussi le transport et l'exportation des produits de la colonie). Les Canadiens se plaignent et prennent même les armes dans les campagnes. La situation se rétablira sous Hocquart. Les abus sont notables surtout sous Bégon et, plus tard, sous Bigot.

La classe supérieure de la colonie, elle aussi, agit selon ses intérêts. Ceux-ci ne s'identifient pas toujours à ceux de la colonie. Atteints par la crise, les seigneurs-négociants étaient portés à pressurer les censitaires. Ceux-ci ignoraient souvent leurs droits de censitaires, et bien des concessions avaient été faites sur la parole des seigneurs ou sur de simples billets. La tentation était facile pour les seigneurs d'étendre, à leur avantage, les obligations des censitaires. Les «Arrêts de Marly » fournirent une garantie à la libre possession des terres par le colon et obligèrent les seigneurs à faire défricher plus rapidement leurs terres. Mais le roi fut très souvent obligé, par la suite, d'en exiger l'application, surtout contre les seigneurs. Le régime seigneurial, dans son ensemble, sert les intérêts du censitaire plus directement que ceux du seigneur. D'ailleurs, dans les périodes de prospérité, les seigneurs ne sont généralement pas tracassiers.

Les difficultés du marché eurent comme autre conséquence d'augmenter le commerce interlope. Depuis plusieurs années déjà, les commerçants canadiens font porter une partie de leurs fourrures à Albany par les Indiens. La crise rend plus importante cette contrebande. Bégon écrit en 1715 qu'il passe tous les ans 
50,000 livres de castor aux Anglais. En 1737, la Compagnie des Indes reçoit 180,000 livres de castor, et Hocquart affirme que la plus grande partie va à Albany. Il est évident que cette pratique nuit au commerce métropolitain alors que la fabrication des chapeaux est redevenue une production florissante. Mais la contrebande est «très profitable aux marchands de Montréal » qui vendent plus cher sur le marché anglais, font deux expéditions par année, sont payés en argent et évitent l'impôt de 25 p. 100 sur le castor. C'est ainsi que la bourgeoisie est parvenue à se dédommager des pertes subies durant la crise.

La Cour tentait bien de contrôler la sortie du castor. Ce fut en vain. Si les commerçants canadiens continuent cette énorme contrebande, c'est qu'ils se protègent entre eux, qu'ils s'entendent avec les autorités coloniales pour rendre inefficaces les ordonnances du roi, et que ce commerce clandestin représente à leurs yeux la liberté du commerce extérieur, et, dans les circonstances particulières du Canada, le droit de vivre. Il nous suffit d'indiquer quel chemin a pris la liberté de groupe des Canadiens dans cette circonstance particulière. Si certaines réglementations métropolitaines étouffent l'activité économique de la colonie, la classe supérieure cherche à faire relâcher l'étreinte. Et les bienfaits de la liberté qu'elle se gagne, grâce à son nombre et à son organisation, retombe sur la collectivité qu'elle entraîne puisque l'ensemble de la population canadienne vit de la traite.

Après avoir tenté de freiner ce mouvement par de multiples ordonnances, Hocquart en vient, en 1743, à écrire que le commerce étranger ne peut nuire à la France. C'était admettre l'inutilité des mesures métropolitaines. Les négociants canadiens, évincés du marché des fourrures après l'effondrement de la Compagnie de la Colonie, avaient réclamé, après la création de la Compagnie d'Occident (1717), la liberté de la traite du castor. En 1720, le roi accorde cette liberté en exigeant des droits sur le produit. Ces arrangements ne satisfont pas les Canadiens. Le roi rétablit le monopole quelques années plus tard, et il en sera ainsi jusqu'à la fin du régime français. On ne réussira jamais à contrôler ce trafic clandestin. Le monopole ne 
rend le commerce interlope que plus prospère. La contrebande, organisée avec finesse et minutie, implique toute la société canadienne qui y participe ou la tolère. Le même commerce clandestin a existé au Cap-Breton. Nous pourrions, de ce fait, dégager les mêmes conclusions, puisque ce sont des membres de la bourgeoisie canadienne qui en sont les entrepreneurs.

Dans le même temps, l'administration coloniale subit des changements qui auraient pu nuire à la bourgeoisie canadienne. Après les «troubles » de 1728, l'intendant perd de son influence et le Conseil, dépossédé de ses attributions politiques, est réduit à une fonction judiciaire. Or, il arrive que Hocquart, nommé, l'année suivante, commissaire général, devient intendant en 1731 et remplit cette fonction pendant vingt ans. Ses qualités personnelles ont restitué à la fonction d'intendant le prestige dont elle jouissait avant 1728. Le Conseil, toutefois, n'a pas eu le même sort. Il n'y reste plus, après ce temps, que des bonnes volontés. La bourgeoisie canadienne y perdait l'instrument officiel de son influence politique.

Mais quelques années auparavant, soit en 1717, les négociants avaient obtenu la permission de s'assembler tous les jours dans les villes de Québec et de Montréal pour y traiter de leurs affaires de commerce. Cette bourse avait le droit de nommer deux de ses membres "pour faire au nom de tous, au gouverneur général et à l'intention de la Nouvelle-France, les représentations ... nécessaires pour le bien de leur commerce». Ainsi, la direction du commerce se trouvait étroitement liée à la direction politique, malgré la diminution du Conseil.

En 1750, le syndic des marchands de Québec s'intitulera syndic «des bourgeois, négociants et habitants de cette ville ». Cette réunion de la classe dirigeante, cette bourse, préconise et soutient «le système canadien » en politique, système dirigée vers la protection du commerce et des territoires qui y sont indispensables. Ce système consistait à attirer les Iroquois dans la sphère d'influence des Canadiens en les divisant et en leur inspirant la terreur des armes canadiennes, sans heurter de front ni les Iroquois, ni les Anglais. Politique d'équilibre, réa- 
liste, amorale et sans justice, si l'on veut, ce système était aussi celui des négociants de fourrures de New York.

Les seigneurs-négociants tiennent à conserver leurs seigneuries. Si les "Arrêts de Marly » ne sont pas appliqués à la satisfaction du roi, c'est que les seigneurs empêchent Hocquart de les appliquer contre eux. Toutefois, en 1741, vingt seigneuries sont réunies au domaine du roi. Il aurait dû y en avoir beaucoup plus, mais le gouverneur et l'intendant ne peuvent se mettre les seigneurs à dos. Cette classe a la protection de la Cour. Elle peut agir et acheter la protection. Mais quand les seigneurs commettent des abus en exigeant de leurs censitaires des corvées qui n'étaient pas incluses dans les contrats de concession, l'intendant veille à ce que ces corvées soient limitées aux exigences du contrat. D'ailleurs, en 1716 , après les premières plaintes à cet égard, Bégon interdit aux seigneurs d'inclure cette clause sous peine de nullité du contrat. Les seigneurs qui avaient stipulé cette clause dans leurs contrats de concession continuèrent d'y obliger les censitaires. Ces corvées, de même que «la corvée du Roy », n'étaient qu'une forme d'imposition. La corvée due au seigneur pouvait être payée en argent; en 1716, Bégon la fixait à vingt sous. Le taux n'était pas abusif. Même si les seigneurs faisaient partie du Conseil et pouvaient éluder certaines ordonnances, le peu de revenus qu'ils pouvaient retirer de leurs seigneuries ne les a jamais dirigés vers cette exploitation, et les a éloignés des abus contre les colons.

Vers 1730 , s'ouvre une ère de prospérité pour la NouvelleFrance. Le commerce extérieur, grâce à la création du marché de Louisbourg, va se composer, dans la proportion d'un tiers, de produits agricoles. La colonisation agricole augmente parallèlement. L'industrie va se développer, dans les limites imposées par le mercantilisme français. L'intervention de l'Etat métropolitain a été la principale cause de cet essor. L'industrie coloniale avait besoin, pour se créer, de l'aide de l'Etat métropolitain. A partir de 1732 surtout, le roi accorde des gratifications. Il en supprime par la suite, mais les subventions du roi prennent toutes les formes. 
Ainsi se développe la construction navale. «La Cour fait lancer des navires de guerre à Québec. Ces contrats permettent d'augmenter l'outillage des chantiers maritimes ». Mais avec l'arrivée de Bigot, une nouvelle classe, non pas de propriétaires de seigneuries, mais de parvenus, va diriger les grandes affaires, les entreprises de guerre. Ces protégés de Bigot se serviront des ateliers royaux pour construire une flotte marchande et se rendre maîtres « du transport maritime et du cabotage ». C'est l'industrie lucrative des temps de guerre. Les Forges de SaintMaurice offrent de même «un exemple typique des entreprises industrielles en économie coloniale ». Quelques bourgeois canadiens, désireux d'exploiter les gisements de fer situés près des Trois-Rivières, forment une compagnie, obtiennent des capitaux de l'Etat métropolitain, grâce à l'appui du gouvernement colonial, et jettent les bases d'un gros établissement. La Compagnie s'endette. L'Etat doit en assumer la régie en 1741. Mais les entrepreneurs coloniaux s'enrichissent.

Si ces entreprises sont possibles, c'est que les entrepreneurs font partie de la classe dirigeante de la société canadienne, qu'ils peuvent faire agir le gouvernement colonial, et que la colonie peut encore compter sur l'Etat fondateur pour en obtenir des fonds. Même si le roi fait de mauvaises affaires, les entrepreneurs s'enrichissent. Ces entreprises profitent en définitive à ceux qui les dominent et, indirectement, à la collectivité canadienne. Cette intervention de l'Etat, qui supplée au défaut d'initiative des individus ou à leur incapacité financière, relève d'ailleurs de la plus pure doctrine libérale en même temps que du mercantilisme.

Le mercantilisme a par ailleurs empêché la création de petites industries. La théorie française du pacte colonial s'illustre bien par le fait suivant: vers 1730, un industriel canadien, appuyé du gouverneur et de l'intendant, demande à la Cour la permission d'établir au Canada une manufacture de chapeaux. Comme il ne fallait pas concurrencer les manufactures du royaume, on lui refusa cette permission. Le mercantilisme d'industrie, en retardant le développement de la colonie, réduit la liberté économique des Canadiens. Nous avons vu qu'il y avait certaines 
compensations, mais ces mesures restent nuisibles, même si le Canada se développe dans les cadres de l'empire français dont il partage la fortune.

Après 1713, nous remarquons donc que, même si la France ne se soucie guère de sa colonie, c'est encore le roi qui solde la plus lourde part des frais de l'administration coloniale, soit environ $70 \mathrm{p}$. 100, et qu'une forte proportion de ces fonds tombe dans les mains des Canadiens. Les sommes que le roi emploie pour reconstruire ou ériger des forts activent l'économie canadienne. Quand des entrepreneurs et des fournisseurs locaux dirigent ces travaux, les dividendes alimentent le commerce de la colonie. Les subventions aux industries ont pris toutes les formes, nous l'avons dit, même si le royaume s'exécute à regret. En somme, les fonds d'Etat sont à la disposition du Canada. Le courant commercial est établi avec la France et la marchandise canadienne y est reçue. Le crédit de la métropole et celui de la colonie sont à la disposition des entrepreneurs canadiens qui agissent et font agir leur gouvernement, et la direction politique est assumée en grande partie par les Canadiens.

Les dérangements provoqués par l'arrivée de Bigot ont pris de l'importance surtout du fait de la guerre totale, la guerre de la Conquête. Bigot est devant un Conseil impuissant. Il va contrôler la manutention des vivres et des fournitures et le transport. Toute l'activité économique va se concentrer entre ses mains. Pour réaliser les bénéfices financiers qu'ils escomptent, Bigot et son groupe doivent modifier la politique canadienne. Le système canadien favorisait le commerce détenu par l'aristocratie canadienne au sud des Grands Lacs. C'est ainsi que Bigot sera amené à dénoncer ce système. Du Quesne arrive en 1753 et lance une campagne contre les Anglais dans l'Ohio. La bourgeoisie canadienne réprouve cette politique non seulement parce que cette campagne lui fait perdre la traite dans cette région, mais parce qu'elle juge cette ligne de conduite téméraire. Même en 1755 , les Canadiens voudront encore mater les sauvages sans provoquer les Anglais. On comprend alors le mécontentement universel des Canadiens. La Cour répond aux vœux des Canadiens en nommant Vaudreuil gouverneur général, mais Bigot se 
défend si bien qu'il revient à son poste. Entouré d'un groupe de parvenus qui lui doivent leur fortune, il dirige la Grande Société qui réunit tous les monopoles, à la faveur de la guerre. Si les Canadiens sont mécontents, c'est qu'ils ont été déplacés et que cette guerre va contre la ligne de conduite, suivie depuis 1713 , qui consistait à exploiter le Centre-Ouest sans attaquer les Anglais. Le conflit devient inévitable: ni les Canadiens ni les Anglais ne veulent être évincés de ce territoire qui est d'une nécessité essentielle à leur expansion.

Si le système canadien représente le moyen, pour les Canadiens, de conserver leur liberté économique et politique, on comprend l'importance qu'ils vont attacher au conflit. L'un des contestants doit soumettre l'autre; les adversaires en sont conscients. La matière de notre sujet s'épuise ici, à la soumission, par la Conquête, du groupe dont nous avons traité.

La liberté que les Canadiens ont possédée leur a permis de concevoir une politique qui visait à dominer le continent nordaméricain. Il y a eu une politique d'inspiration canadienne: le système canadien existait dès avant 1700 . Le commerce intérieur a été entre les mains des Canadiens de même qu'une bonne partie du commerce extérieur. Si la métropole était encore, au moment de la Conquête, nécessaire au développement harmonieux du Canada, ce fait n'empêchait pas que le Canada fût un Etat. Les individus qui le composaient avaient conscience d'appartenir à une même collectivité. Dès 1690 , le sentiment national avait uni les Canadiens au-dessus des particularismes régionaux. Ce peuple était conscient d'être un groupe ethnique distinct. Si les Canadiens désiraient tant Vaudreuil, c'est que ce dernier personnifiait la bourgeoisie canadienne, qu'il s'identifiait aux Canadiens comme ceux-ci s'identifiaient à lui. Vaudreuil est impérialiste, mais, dans les cadres de l'empire, il travaille à «renforcer les structures sociales et l'armature politique du Canada ».

Mais le degré d'évolution où est parvenue la société canadienne est encore le témoignage le plus éloquent sur l'existence de la liberté économique et politique des Canadiens sous le régime français. Si la civilisation exprime un degré de développement plus ou moins avancé de vie politique, sociale, économique et 
intellectuelle dans un groupement organisé, nous constatons qu'en 1750 il ne manque rien d'essentiel à la civilisation canadienne. Elle présente toutes les caractéristiques d'une civilisation occidentale. Elle a été complète et originale, à la mesure du peuple qui l'a créée pour le servir. Une colonie ne peut constituer un tout aussi organique et organisé sans la direction d'une classe supérieure. Cette classe supérieure n'aurait pas existé au Canada si elle n'avait eu la liberté de s'y créer une patrie et d'y jouer son rôle de dirigeante. L'aboutissement final de cette croissance est encore plus révélateur que le nombre des faits répétés, et il confère à ces faits une éloquente signification.

\section{Rosario BILODEAU,}

Docteur ès lettres de l'Université de Montréal, Professeur au Collège militaire royal de Saint-Jean, P.Q. 